\title{
A Parametric Study of Inductive SWIPT Systems Assisted by Metamaterial Using Virtual Magnetic TL-Based Channel Modeling
}

\author{
Jorge Virgilio de Almeida ${ }^{1}$ (), Eduardo Costa da Silva ${ }^{1}$ (), Marbey Manhães Mosso ${ }^{1}$ (), \\ Carlos Antonio França Sartori ${ }^{2,3}$ (1) \\ ${ }^{1}$ Dept. of Electrical Engineering, Pontifical Catholic University of Rio de Janeiro, Rio de Janeiro, RJ 22430- \\ 060, Brazil \\ jorgevirgilio21@gmail.com,edusilva@ele.puc-rio.br,marbeyg@gmail.com \\ ${ }^{2}$ Dept. of Electrical Energy and Automation Engineering, Polytechnic School of University of São Paulo, São \\ Paulo, SP 05508-010, Brazil \\ ${ }^{3}$ Nuclear and Energy Research Institute, University of São Paulo, São Paulo, SP 03178-200, Brazil \\ sartori@pea.usp.br
}

\begin{abstract}
This paper presents a general methodology based on the description of the inductive channel as virtual magnetic transmission-lines (VMGTLs). In comparison with other existing methods, VMGTL approach presents a better physical insight of the channel behavior since the model correctly preserves the energy flow between the transmitting and receiving coils. Besides that, it facilitates the integration into the analysis of highly nonlinear and dispersive structures such as metamaterial (MTM) lenses. Particularly, the virtual-TL analogy clarifies that the enhancement of the transmission gain between any two coils assisted by MTM is not due to an enhanced coupling between the drivers, as usually claimed, but to the emergence of propagating near-field modes supported by the MTM. This approach, by means of a parametric study, also indicates, for the first time, that MTMs could be employed not only for the increasing of power but also of data transfer due to the emergence of a sub-resonant region of minimum distortion. Nonetheless, since both effects are mutually exclusive, no passive MTM structure could simultaneously improve power and data transmission.
\end{abstract}

Index Terms - SWIPT, IoT, inductive channel, metamaterial, virtual magnetic transmission lines.

\section{INTRODUCTION}

Inductive-coupling based systems have shown to present enormous advantages over any other power and data transmission mechanisms like electromagnetic (EM) or acoustic waves in extreme environments, such as underground or underwater, once the vast majority of the materials in the Universe are magnetic insensitive (that is to say, their permeability is the same of free-space). Because of their strong material dependency, acoustic waves present low power density, extremely high latency and multipath problems, which makes them unsuitable for any reliable mission-critical or real-time Simultaneous Wireless Information and Power Transfer (SWIPT), while propagating EM modes tend to attenuate very quickly in such lossy media, as a consequence of their usually high conductivity and/or high permittivity values. For these reasons, the envisioned Internet of Underwater 
and Underground Things (IoUT) is expected to primarily rely on inductive channels [1] - [3].

Despite their virtues, however, the fundamental flaws of these magnetic links remain a challenge for designers. Particularly, their limited range of operation and their strictly nonlinear nature are still huge drawbacks to systems based on inductive coupling. In the last decades, many different techniques have been proposed trying to mitigate such problems. Concerning the limitations in the operating range, the main strategies employed by designers are: resonant relays [4]- [6] or near-field MTM based lenses [7] - [9].

These two strategies are actually very similar to each other since both employ highly frequencyselective circuits (high-Q resonators) to compensate the magnetic coupling decaying. According to [10], the MTM solution differs from the previous one due to its "lattice gain", which emerges from the collective behavior of the unit cells. In both cases, however, the common-used analytical method consists of solving a set of linear equations relating the circuit component of the drivers (resistance, inductance, capacitance, etc.), which can be quite complex to solve, particularly when MTM artificial crystals made out of many small resonant coils - are involved. Also, there is a fundamental problem in taking this approach, since, as it will be further shown here, coupling is not the unique power transfer mechanism of the near field. This oversimplification usually conducts to the wrong conclusion that assumes signal variance and power to be the same thing in SWIPT. It is a core problem for these systems since their control and tuning depends on the knowledge of the channel.

Some papers have tried to overcome this problem by characterizing the inductive channel directly through measurement [11]. Yet this strategy is also quite complicated to be implemented, especially considering operating scenarios like massive MIMO-SWIPT. Based on that, a more systematic inductive channel modeling technique based on virtual magnetic transmission line (VMGTL) is proposed, aiming to solve large-scale systems. The model was recently introduced in [12] as an extension of the theory of the magnetic TLs for non-confined magnetic flux. Like the GyratorCapacitor Lumped Element Model [13], VMGTL also correctly preserves the representation of the energy flow along the inductive channel, with the advantage of being a distributed model.

In this paper, VMGTL-based channel modeling is employed to evaluate the impact of the insertion of MTM lenses in the magnetic link. In section II, the VGMTL-based channel modeling is briefly introduced. In Section III, the proposed model is employed in a parametric study in order to clarify the inductive channel response to MTM-based artificial magnetic conductors. In section IV, the obtained results are used to demonstrate that MTM-assisted inductive channels transfer power primarily by means of backward propagating modes at their resonance frequency and that MTM also created a region of minimum-distortion at the sub-resonant region appropriated for data transmission. The main results obtained using the VGMTL model are validated by numerical simulations. 


\section{CHANNEL MODELING USING A VMGTL APPROACH}

In this section, VMGTL technique is presented. This theoretical development is the same one previously introduced in [12].

\section{A. Inductive channel modeling using VMGLT}

The main design principle of the VMGTL model is the observation that electric/magnetic fluxes and charges have the same dimension. Hence, any time-varying flux behaves as a "virtual current", which are better known as displacement magnetic/electric currents:

$$
\begin{aligned}
j \omega \phi_{m} & =j \omega \iint \boldsymbol{B} \cdot d \boldsymbol{s}=\iint j \omega \boldsymbol{B} \cdot d \boldsymbol{s}=\iint \boldsymbol{J}_{m, d} \cdot d \boldsymbol{s}=I_{m, d} \quad[V] \\
j \omega \phi_{e} & =j \omega \iint \boldsymbol{D} \cdot d \boldsymbol{s}=\iint j \omega \boldsymbol{D} \cdot d \boldsymbol{s}=\iint \boldsymbol{J}_{e, d} \cdot d \boldsymbol{s}=I_{e, d} \quad[A]
\end{aligned}
$$

Like real currents, displacement ones can also guide EM fields, as illustrated in Fig. 1.

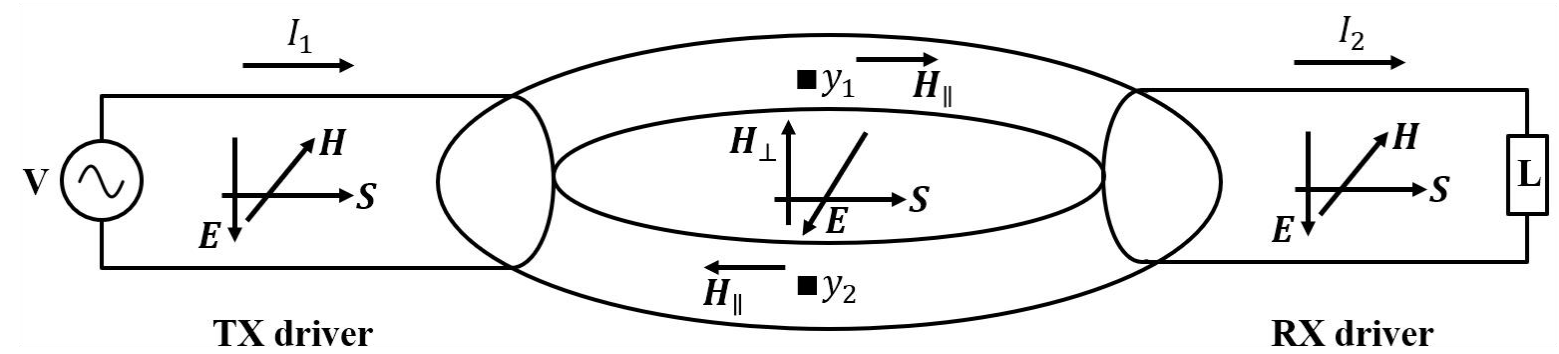

Fig. 1. The displacement magnetic current $I_{m, d}$ between the transmitting and receiving coils forms a virtual circuit similar to a conventional TL. According to Poynting theorem, power flow in the inductive channel depends on the perpendicular component of the magnetic near field created by the magnetic potential difference between the flux in positions $y_{1}$ and $y_{2}$.

For all purpose, a VMGTL consists of two insulated drivers with no physical charge flowing between them and interacting only by means of the non-confined magnetic flux. The driving force of a VMGTL is the magnetic potential $V_{m}$ given by:

$$
V_{m}=\int_{y_{1}}^{y_{2}} \boldsymbol{H}_{\perp}(\omega) \cdot d \boldsymbol{I} \quad[A]
$$

The magnetic potential $V_{m}$ and the magnetic displacement current $I_{m, d}$ of the VMGTL follow propagation equations analogous to the ones of conventional TLs:

$$
\begin{gathered}
\frac{d}{d z} V_{m}(z)=\left(\frac{R_{m}^{\prime}}{j \omega}+G_{e}^{\prime} K_{m}^{2}+j \omega C^{\prime} K_{m}^{2}\right) I_{m, d}(z)=Z_{m} I_{m, d}(z) \quad\left[\frac{A}{m}\right] \\
\frac{d}{d z} I_{m, d}(z)=\left(G_{m}^{\prime} K_{m}^{2}+j \omega L^{\prime} K_{m}^{2}\right) V_{m}(z)=Y_{m} V_{m}(z) \quad\left[\frac{V}{m}\right]
\end{gathered}
$$

where $Z_{m}, Y_{m}, R_{m}^{\prime}, K_{m}, G_{e}^{\prime}, G_{m}^{\prime}, L^{\prime}$ and $C^{\prime}$ denote the series magnetic impedance, the shunt magnetic admittance, the magnetic reluctance, the magnetic coupling coefficient, the electrical conductance, the magnetic conductance, the inductance and the capacitance per unit length, respectively.

From Eqs. 4-5, it can be seen that when $K_{m} \rightarrow 1$ the TL analogy becomes quite similar to the 
magnetic TLs proposed by [14]. However, VMGTL approach keeps its validity even when $K_{m}$ is low until the limiting case where $Y_{m} \rightarrow 0$.

The characteristic electrical impedance $Z_{0}$ of the VMGTL, its complex propagation parameter $\gamma$ and its load reflection coefficient $\Gamma$ are defined as:

$$
\begin{gathered}
Z_{0}=\frac{I_{m, d}(z)}{V_{m}(z)}=\sqrt{\frac{Y_{m}}{Z_{m}}}=\sqrt{\frac{G_{m}^{\prime} K_{m}^{2}+j \omega L^{\prime} K_{m}^{2}}{R_{m}^{\prime}+G_{e}^{\prime} K_{m}^{2}+j \omega C^{\prime} K_{m}^{2}}} \quad[\Omega] \\
\gamma=\sqrt{Z_{m} Y_{m}}=\sqrt{\left(\frac{G_{m}^{\prime} K_{m}^{2}}{j \omega}+L^{\prime} K_{m}^{2}\right)\left(-\frac{R_{m}^{\prime}}{\omega^{2}}+\frac{G_{e}^{\prime} K_{m}^{2}}{j \omega}+C^{\prime} K_{m}^{2}\right)} \quad\left[\frac{r a d}{m}\right] \\
\Gamma=\frac{Z_{R X}-Z_{0}}{Z_{R X}+Z_{0}}
\end{gathered}
$$

where $Z_{R X}$ is the load at the receiver. The general solution of this one-dimensional Helmholtz problem is [15]:

$$
\begin{array}{cc}
I_{m}(z)=I_{m}(0) e^{-\gamma z}\left(1+\Gamma e^{2 \gamma z}\right) & {[V]} \\
V_{m}(z)=\frac{I_{m}(0)}{Z_{0}} e^{-\gamma z}\left(1-\Gamma e^{2 \gamma z}\right) & {[A]}
\end{array}
$$

The total power flow at any point of the inductive channel will then be:

$$
P_{f}(\omega)=V_{m} I_{m, d}^{*} \quad[W]
$$

By definition, the frequency-domain channel transfer function of the system is given by:

$$
H(\omega)=\frac{V_{m}(0)}{V_{m}(-l)}=\frac{e^{-\gamma D}(1-\Gamma)}{\left(1-\Gamma e^{-2 \gamma D}\right)}
$$

where $D$ is the separation distance of the drivers.

Notice that the model validity is limited to $D>r / 2$, where $r$ is the radius of TX and RX coils.

\section{B. Estimation of the model parameters}

To determine the five parameters presented in Eqs. 4-5, some simplifying hypotheses must be made. The first hypothesis assumes that the distributed elements $G_{e}^{\prime}, G_{m}^{\prime}, L^{\prime}$ and $C^{\prime}$ are uniform throughout the inductive channel. If so, they can be directly estimated as follows:

$$
\begin{gathered}
L^{\prime}=\frac{L_{0}}{D} \quad\left[\frac{H}{m}\right] \\
C^{\prime}=\frac{C_{0}}{D} \quad\left[\frac{F}{m}\right] \\
G_{e}^{\prime}=\omega C^{\prime} \frac{\operatorname{Im}\left\{\varepsilon_{r}\right\}}{\operatorname{Re}\left\{\varepsilon_{r}\right\}} \quad\left[\frac{S}{m}\right] \\
G_{m}^{\prime}=\omega L^{\prime} \frac{\operatorname{Im}\left\{\mu_{r}\right\}}{\operatorname{Re}\left\{\mu_{r}\right\}} \quad\left[\frac{\Omega}{m}\right]
\end{gathered}
$$

where $L_{0}$ and $C_{0}$ are the equivalent inductance and capacitance of the TX coil, and $\varepsilon_{r}$ and $\mu_{r}$ are the relative permittivity and permeability of the channel medium. 
However, the estimation of distributed $R_{\mathrm{m}}^{\prime}$ is a little more complicated since the original concept of magnetic reluctance $R_{\mathrm{m}}$ was conceived for magnetic circuits where most of the magnetic flux is confined into a ferromagnetic core with constant transverse section.

First, we observe that, by definition, $R_{\mathrm{m}}$ is given by:

$$
R_{m} \triangleq \frac{V_{m}}{\phi_{m}} \quad\left[H^{-1}\right]
$$

where $V_{m}$ and $\phi_{m}$ are the TX-coil magneto-motive force of the transmitter and the mutual flux between the TX and the RX.

Secondly, we recall that the mutual inductance $M_{R X-T X}$ between the drivers is given by:

$$
M_{R X-T X}=\frac{\phi_{m}^{R X-T X}}{V_{m}^{T X}} \quad[H]
$$

Hence, based on Eq. 17 and Eq. 18, $R_{m}^{\prime}$ can be approximated by:

$$
R_{\mathrm{m}}^{\prime} \propto \frac{1}{M^{R X-T X} \xi} \quad\left[H^{-1}\right]
$$

where $\xi$ is dimensionless geometrical weighting parameter.

Making another simplifying hypothesis that $\xi$ is estimated by the square of the radius of the coils

$$
\xi \sim r^{2}
$$

the equivalent VMGTL of the system is now completely characterized. It is important to notice that the model assumes that $R_{\mathrm{m}}^{\prime}$ is constant and not a function of the spatial coordinates, thus the approximation will become less and less accurate if $D / r$ increases, particularly concerning the estimation of $\gamma$. Another limitation is that the model is valid only if the TX and RX coils are electrically small and far from their resonance frequency (this is, $r \ll \lambda / 2$ ).

\section{MTM interaction with the Inductive Channel}

As demonstrated in [12], the effects of the MTM on the inductive channel can be introduced to the VMGTL model by defining an equivalent impedance of the MTM.

Assuming MTM slabs made of a constant lattice of spiral resonators (SRs) unit cells, the equivalent distributed $Z_{M T M}^{\prime}$ is found to be:

$$
Z_{M T M}^{\prime}(\omega)=j \omega \mu_{0}\left(1+\operatorname{sign}(F) \frac{|F| \omega^{2}}{\omega_{0}^{2}-\omega^{2}-j \omega \omega_{0} / Q}\right) \quad\left[\frac{\Omega}{m}\right]
$$

where $\omega_{0}, F$ and $Q$ are its resonance angular frequency, the coupling coefficient between adjacent cells of the lattice and the quality factor of the unit cells, respectively.

Hence, Eq. 5 can be rewritten as

$$
\begin{aligned}
\frac{d}{d z} I_{m, d}(z)= & \left(G_{m}^{\prime} K_{m}^{2}+j \omega L^{\prime} K_{m}^{2}+Z_{M T M}^{\prime}(\omega) K_{m, M T M}^{2}\right) V_{m}(z) \\
& =Y_{m} V_{m}(z) \quad\left[\frac{V}{m}\right]
\end{aligned}
$$


since the MTM changes the variation of the magnetic flux with the distance. In other words, the MTM alters the magnetic admittance $Y_{m}$ of the channel.

The magnetic coupling between the MTM and the TX and RX coils depends on the physical size of the MTM. Assuming a semi-infinite MTM plane in the transversal section of the channel, it can be approximated by:

$$
K_{m, M T M} \cong 2 K_{m}
$$

This simplifying hypothesis can be assumed if the MTM dimensions are at least four times larger than TX and RX coils radius $r$.

\section{MODEL APPLICATION AND VALIDATION}

The considered system is composed of two electrically small far-from-resonance coils separated by a fixed distance $D=15 \mathrm{~cm}$. The coils are made of copper with diameter $p=1 \mathrm{~mm}$. The selfinductance $L_{0}$ of the small circular loop is found in closed form in literature [16]. Their magnetic coupling coefficient $K_{m}$ can be estimated using the thin-wire approximation [17]. In the present work, all the MTM slabs are identical to the one employed in [12], with the same equivalent $Z_{M T M}^{\prime}$. Also, they are supposed to be placed perpendicularly to the magnetic flux.

To demonstrate the model's accuracy, the analytical channel estimation is compared in Figs. 2-3 with the one obtained on Keysight ADS2020 using the Method of Moments. Some discrepancies between the analytical and the numerical results exist as expected due to the simplifying hypotheses applied to Eqs. 19-23.

As shown in Fig. 4, when there is no MTM in the channel, the imaginary part of the propagation constant $\gamma$, known as the phase constant $\beta$, is null, and the real part of $\gamma$, known as the attenuation constant $\alpha$, is low-varying with frequency. In this situation, the only mechanism of power exchange between the electric interfaces is their mutual coupling. The H-field lines generated by each driver are in contact with its neighbor, hence inducing current on it according to Faraday's Law. This case can be completely characterized by means of the mutual inductance matrix and similar circuital analogies.
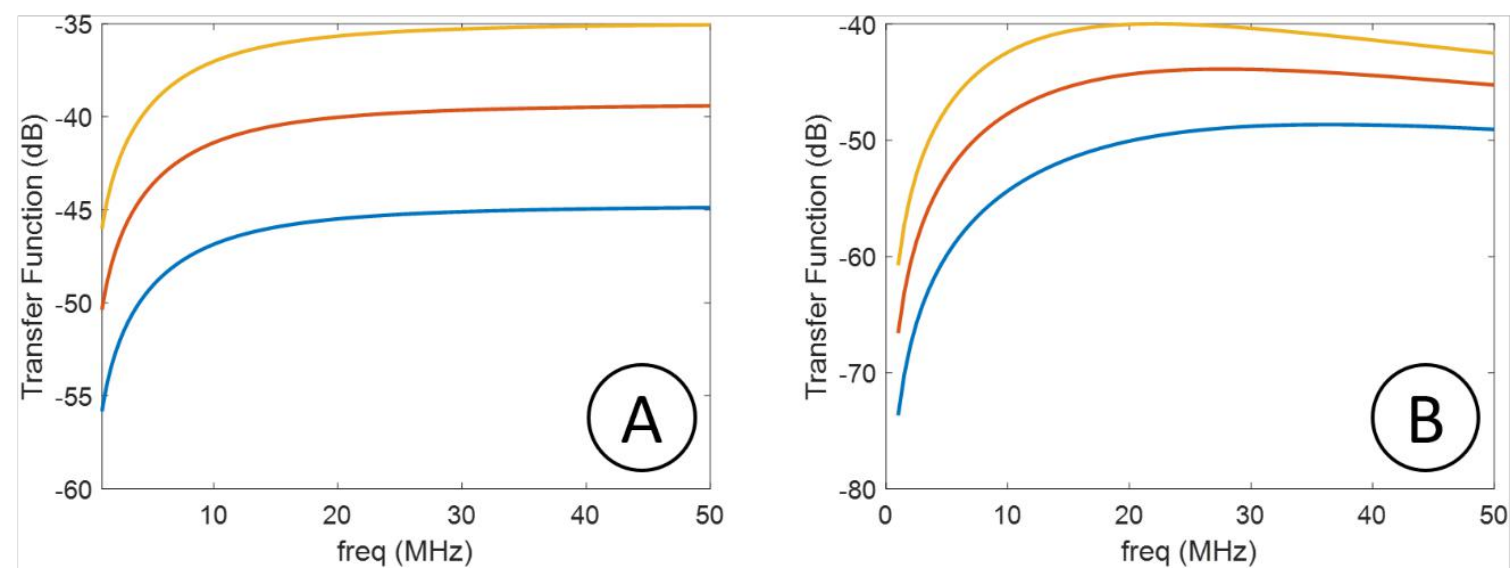

Fig. 2. Magnitude of the transfer function as a function of frequency for a VGMTL without MTM: analytical model (a) and numerical simulation (b) for $\mathrm{r}=4 \mathrm{~cm}$ (blue), $\mathrm{r}=5 \mathrm{~cm}$ (orange) and $\mathrm{r}=6 \mathrm{~cm}$ (yellow). 

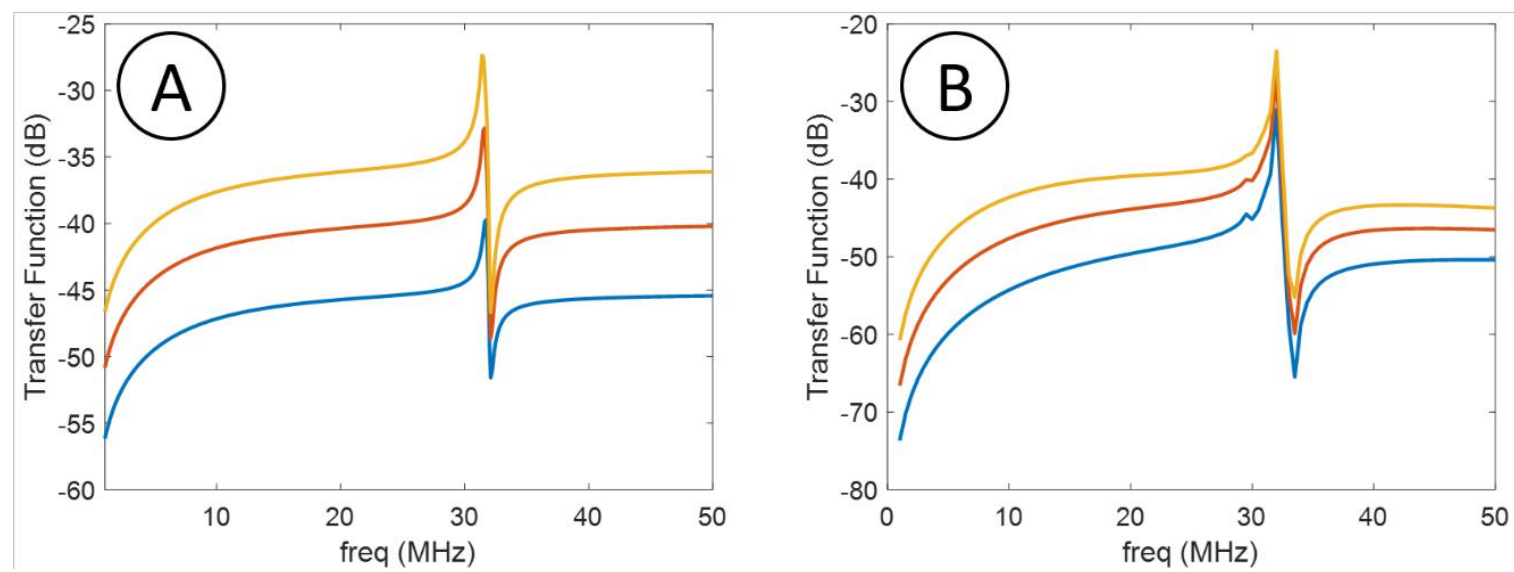

Fig. 3. Magnitude of the transfer function as a function of frequency for a VGMTL assisted by one MTM slab: analytical model (a) and numerical simulation (b) for $r=4 \mathrm{~cm}$ (blue), $r=5 \mathrm{~cm}$ (orange) and $r=6 \mathrm{~cm}$ (yellow).
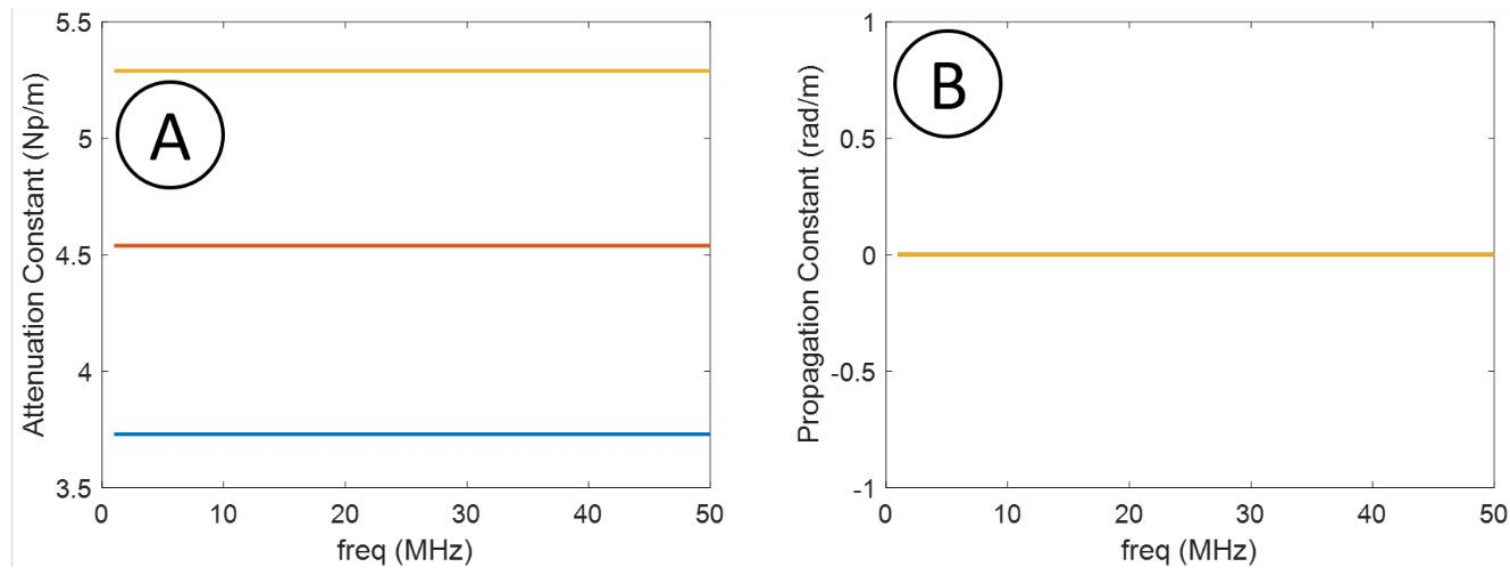

Fig. 4. Attenuation (a) and propagation (b) constants as a function of frequency for a VGMTL without MTM for $r=4 \mathrm{~cm}$ (blue), $r=5 \mathrm{~cm}$ (orange) and $r=6 \mathrm{~cm}$ (yellow).
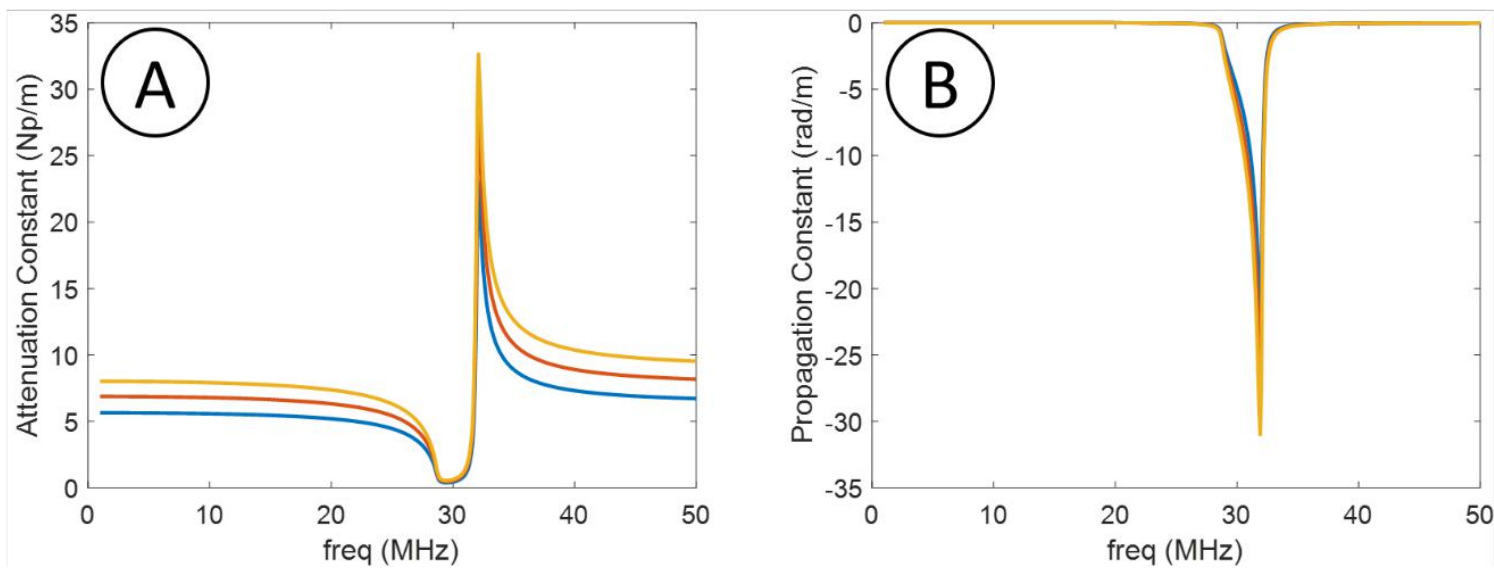

Fig. 5. Attenuation (a) and propagation (b) constants as a function of frequency for VGMTL assisted by one MTM slab for $r=4 \mathrm{~cm}$ (blue), $r=5 \mathrm{~cm}$ (orange) and $r=6 \mathrm{~cm}$ (yellow).

However, if a MTM slab is introduced in the channel, independently of its relative position inside the link, other power transfer mechanism arises. In Fig. 5, the enhancement of the transmission gain around the MTM resonance frequency $f_{0}$ is basically due to the appearance of a passband (non-null propagation constant) in the inductive link. So, the additional power being transferred between the 
drivers is not coming from the enhancement of the coupling, but by propagating modes supported by the MTM. Travelling magnetic waves were already identified and employed in literature in different contexts [18] , but they were never shown to be the very cause of the "enhanced coupling" phenomenon. Nevertheless, this work clearly shows that the additional power being delivered to the receiver in the MTM gain region is not due to any stronger interaction of the evanescent modes but by the emergence of travelling ones.

Evanescent modes are characterized by $\alpha>0$ and $\beta=0$. The greater $\alpha$ becomes, the faster the modes attenuate with distance, diminishing the probability that any coupling will occur between the drivers. If the power gain mechanism was related to the enhancement of the evanescent modes, $\alpha$ should be reduced. Still, as shown in Fig. 5.a, in the region of minimum $\alpha$, the power gain is not maximum (actually, it is negligible in comparison to the case where no MTM slab is employed). As shown in Fig. 5.b, the maximum power gain occurs, in fact, at the frequency where the MTM maximizes both the module of $\alpha$ and the module $\beta$. Based on this, it can be said that the coupling is strongly reduced by MTMs (whose losses increases $\alpha$ ) and that the observed transmission gain occurs primarily due to the appearance of propagating modes $(\beta \neq 0)$. Since the propagating modes transfer power more efficiently than the evanescent ones, MTM-added losses are fully compensated by the power of the magnetic waves and the system experiences a net gain. Notice that since $\beta<0$ these waves are backwardly oriented ones.

Another important fact, totally neglected on MTM-enhanced coupling theory and that becomes clear with VMGTL approach, is that MTMs induce not only a region of high gain but also a passband region with minimum attenuation. This region lies in the sub-resonant region and has a relative flat response in comparison with the region of strong power gain around $f_{0}$. The flatness of this region imposes low distortion to signals and could be used for improved data transmission. Nonetheless, since MTM power gain increases with the module of $\beta$ and since $\beta$ decreases rapidly out of the resonance, the average power transfer is significantly smaller than the peak gain.

This means that MTMs can be used for maximizing either data rate or power transfer, but optimum values for both cannot be simultaneously obtained. Optimum bandwidth-gain trade-off in SWIPT systems lies fundamentally in-between these two cases.

\section{PARAMETRIC STUDY OF A MTM-ASSISTED VMGTL}

\section{A. Channel response as a function of the MTM's $F$ and $Q$ parameters}

As seen in Figs. 6-8, the sensibility of the channel to $F$ is much stronger than to $Q$. While $F$ can be employed to control the bandwidth of the passband region created by the MTM, $Q$-factor can be used to regulate the magnitude of the response. 


\section{B. Channel response as a function of the number of MTM slabs}

Concerning the number of MTMs, Figs. 9-10 show that the peak gain of the high-gain region (strong $\beta$ response) and the minimum-distortion band (flat $\alpha$ response) starts to saturate with the increasing number of MTM slabs inserted into the inductive channel (in this work, the slabs are supposed to be equally distant from each other inside the link). Besides that, at some point, when the separation distance between the MTMs becomes small (lower than the lattice periodicity) due to the increasing number of MTMs, the mutual coupling between the slabs forces them to operate as one single structure, at a much lower $f_{0}$ (see Fig. 10). When the MTMs get coupled, $f_{0}$ is decreased by a factor of $\sqrt{N}$, where $N$ is the number of MTM slabs.
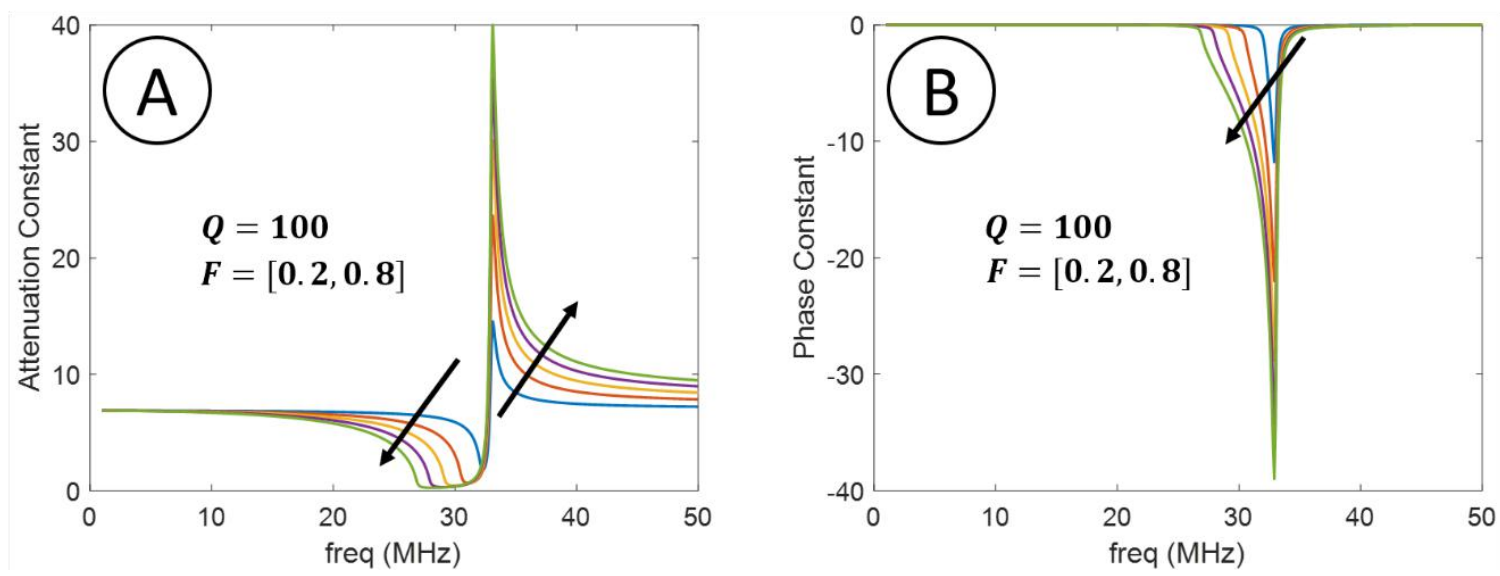

Fig. 6. Sensibility of VGMTL attenuation (a) and propagation (b) constants to $F$.
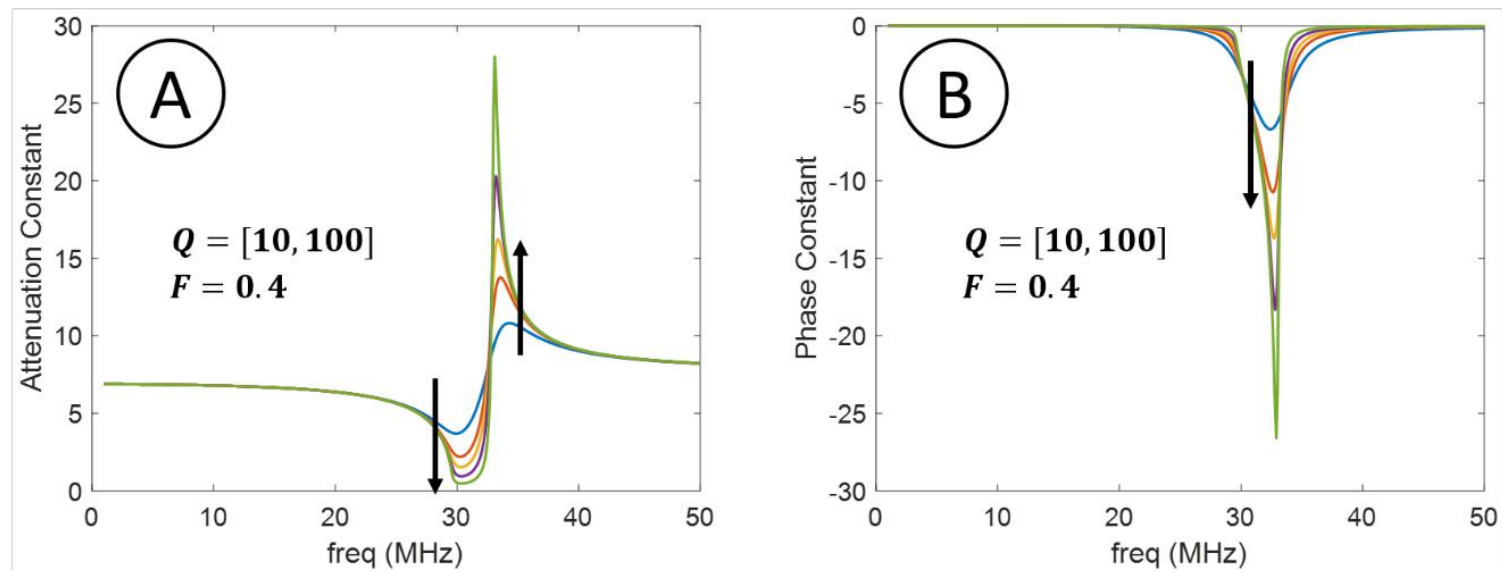

Fig. 7. Sensibility of VGMTL attenuation (a) and propagation (b) constants to $Q$. 

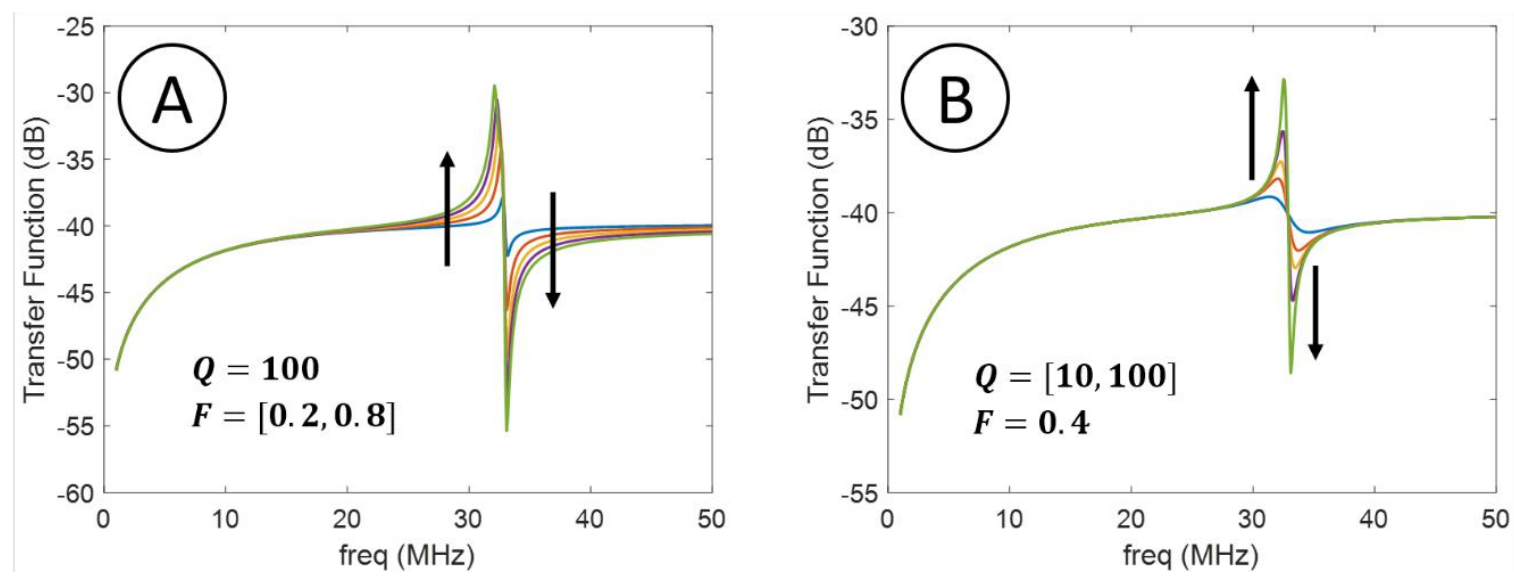

Fig. 8. Sensibility of VGMTL transfer function to $F$ (a) and $Q$ (b). While $F$ governs the bandwidth of the passband, $Q$ regulates only the magnitude of the response.

As shown in Fig. 9, the achievable bandwidth in the minimum-distortion region is much higher (at least one order of magnitude) than the one obtained in the high-gain one but with almost negligible power gain. Conversely, the high-gain region permits delivering much more power to the receiver but at the cost of a very narrow bandwidth. Notice that bandwidth is dramatically reduced in both scenarios when the mutual coupling between the MTMs forces $f_{0}$ to decrease.
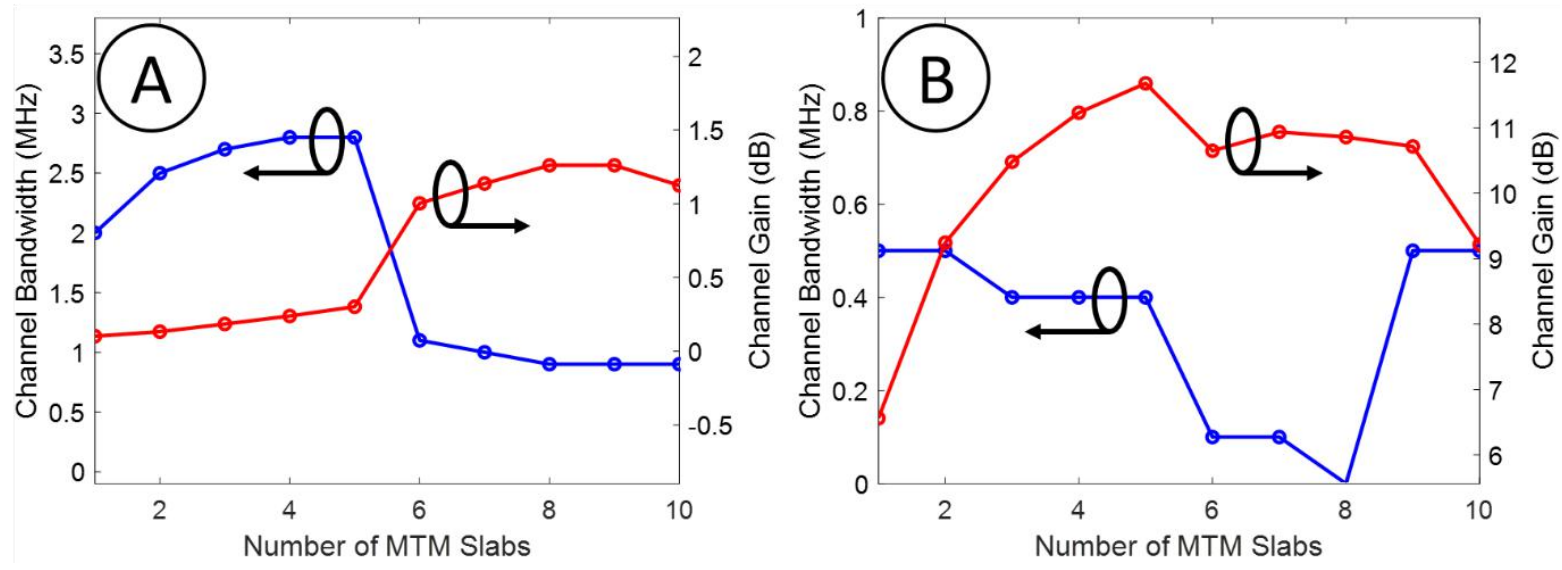

Fig. 9. The bandwidth-gain trade-off when operating at the region of minimum distortion (a) and high gain (b). 


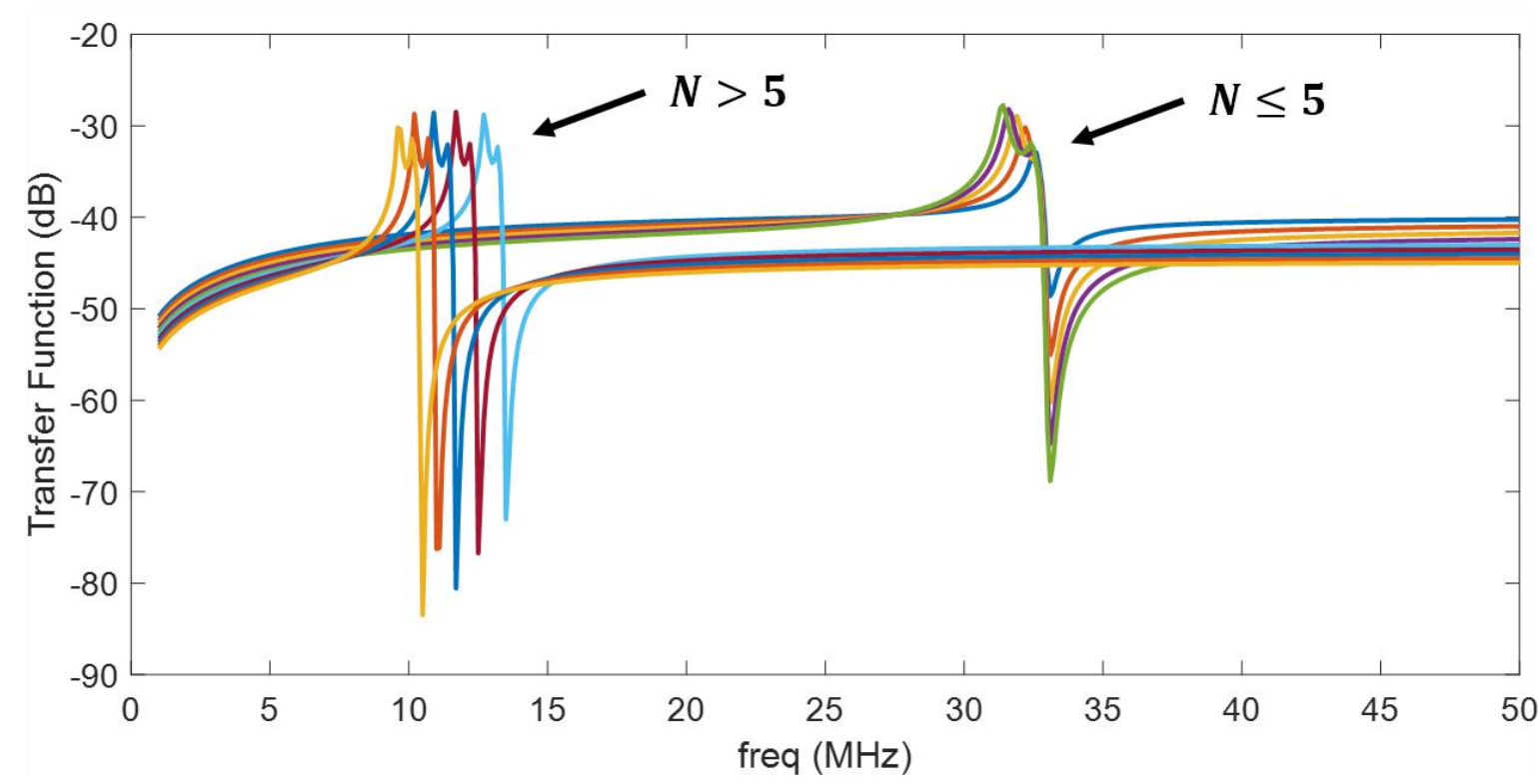

Fig. 10. VGMTL transfer function with an increasing number $N$ of MTM slabs in the channel.

\section{Channel Response to multiple MTMs set to different resonance frequencies}

One possible strategy to increase the capacity of the inductive channel keeping a net power gain would be to use multiple MTM slabs set to different values of $f_{0}$. As shown in Fig. 11 and Fig. 13.a, it permits creating a population of narrow passband regions inside the inductive channel. However, this approach meets its limitation if the MTMs are resonating in immediately adjacent bands since the high gain region of the slabs (this is, maximum $|\beta|$ ) is always followed by an extremely low one due to the anti-resonance phenomenon. In such cases, as shown in Fig. 12 and Fig. 13.b, the peak of one MTM will "fall" into the valley of its neighbor.
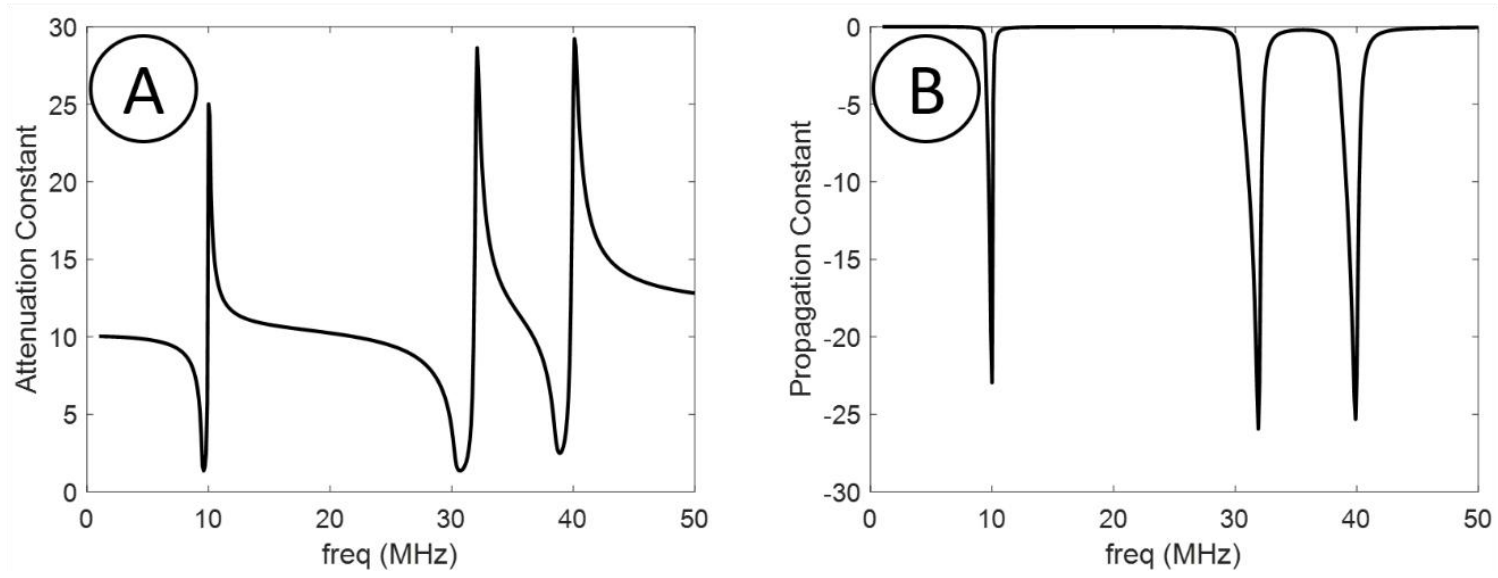

Fig. 11. Attenuation (a) and propagation constants (b) with three MTM slabs for case 1: $f_{01}=10 \mathrm{MHz}, f_{02}=$ $32 \mathrm{MHz}$ and $f_{03}=40 \mathrm{MHz}$. 

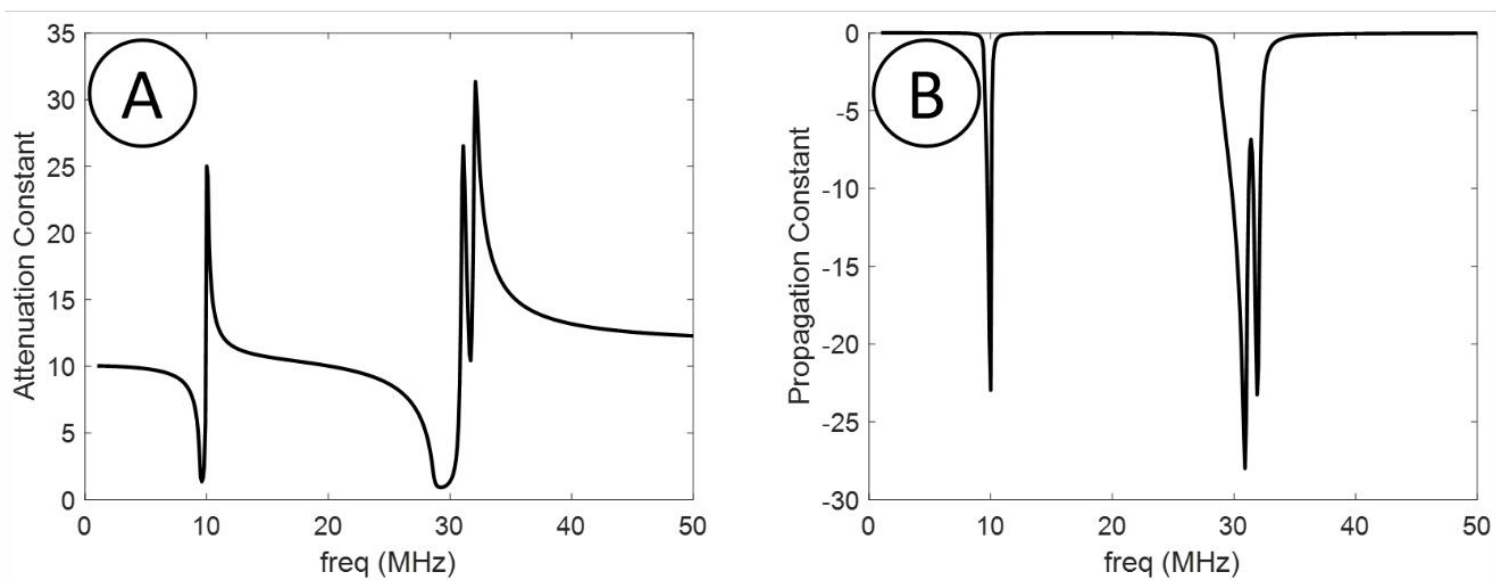

Fig. 12. Attenuation (a) and propagation constants (b) with three MTM slabs for case 2: $f_{01}=10 \mathrm{MHz}, f_{02}=$ $31 \mathrm{MHz}$ and $f_{03}=32 \mathrm{MHz}$.
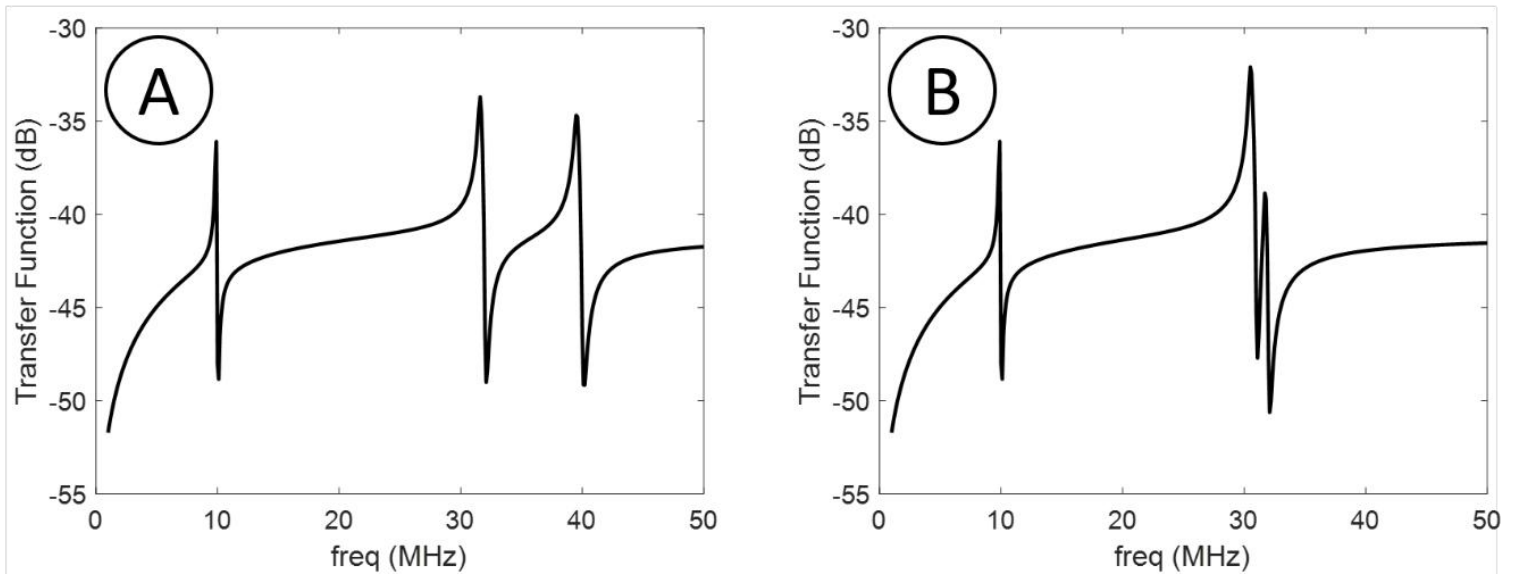

Fig. 13. Results for the VGMTL transfer function with 3 MTM slabs for case 1 (a) and case 2 (b).

\section{CONCLUSION}

In this work, a parametric study of MTM-assisted inductive channels was presented. The channel modeling employed the VMGTL approach in order to clarify the MTM mechanism of power enhancement. Based on this model, MTMs are shown to improve power transmission between the drivers around the resonance not by means of enhanced coupling but backward propagating modes. Besides that, the same approach was able to reveal, for the first time, the existence of a minimumdistortion response in the sub-resonant region of the system that could be exploited to maximize the data rate. However, this region presents just a slight net power gain in comparison with the case where the inductive channel is assisted by no MTM. It implies that MTM-assisted SWIPT must maximize either power or data transfer. Nonetheless, this limitation could be overcome by means of active MTMs with electronically controlled unit-cells capable of switching from one region to the other (high gain or minimum distortion) according to the system needs, establishing reliable self-powered networks in extreme environments such as the foreseen Internet of Underwater Things (IoUT). Finally, in a future work, the authors intend to extend the VMGTL approach to MIMO-SWIPT scenarios. 


\section{REFERENCES}

[1] K. M. Awan, P. A. Shah, K. Iqbal, S. Gillani, W. Ahmad and Y. Nam, "Underwater Wireless Sensor Networks: A Review of Recent Issues and Challenges," Wireless Communications and Mobile Computing, vol. 2019, no. 6470359, p. 20, 2019.

[2] R. Guida, E. Demirors, N. Dave, J. Rodowicz and T. Melodia, "An Acoustically Powered Battery-less Internet of Underwater Things Platform," in 2018 Fourth Underwater Communications and Networking Conference (UComms), Lerici, Italy, 2018.

[3] N. Khalil, M. R. Abid, D. Benhaddou and M. Gerndt, "Wireless sensors networks for Internet of Things," in 2014 IEEE Ninth International Conference on Intelligent Sensors, Sensor Networks and Information Processing (ISSNIP), Singapore, Singapore, 2014.

[4] D. C. Corrêa, U. C. Resende and F. S. Bicalho, "Experiments With a Compact Wireless Power Transfer System Using Strongly Coupled Magnetic Resonance and Metamaterials," IEEE Transactions on Magnetics, vol. 55, no. 8, 2019.

[5] D. Ahn, M. Kiani and M. Ghovanloo, "Enhanced Wireless Power Transmission Using Strong Paramagnetic Response," IEEE Transactions on Magnetics, vol. 50, no. 3, 2014.

[6] F. Zhang and M. Sun, "Efficient Wireless Power Transfer based on Strongly Coupled Magnetic Resonance," in Wireless Power Transfer, 2 ed., River Publishers, 2016, pp. 73 - 104.

[7] B. Wang, W. Yerazunis and K. Teo, "Wireless Power Transfer: Metamaterials and Array of Coupled Resonators," Proceedings of the IEEE, 2013.

[8] Y. Urzhumov and D. R. Smith, "Metamaterial-enhanced coupling between magnetic dipoles for efficient wireless power transfer," Phys. Rev. B, vol. 83, no. 205114, pp. 1 - 10, 2011.

[9] P. Sharma, D. Bhatia and R. S. Meena, "Metamaterial enhanced magnetization induced communication for wireless applications," in 2017 International Conference on Information, Communication, Instrumentation and Control (ICICIC), Indore, India , 2017.

[10] J. V. de Almeida and R. S. Feitoza, "Metamaterial-Enhanced Magnetic Coupling: An Inductive Wireless Power Transmission System Assisted by Metamaterial-Based Mu-Negative Lenses," IEEE Microwave Magazine, vol. 19, no. 4, pp. $95-100,2018$.

[11] T. Arakawa, S. Goguri, J. V. Krogmeier, A. Kruger, D. J. Love, R. Mudumbai and M. A. Swabey, "Optimizing Wireless Power Transfer FromMultiple Transmit Coils," IEEE Access, vol. 6, pp. 23828 - 23838, 2018.

[12] J. V. de Almeida, G. L. Siqueira, M. M. Mosso and C. A. F. Sartori, "Mu Negative Metamaterials Seen as Band Limited Non Foster Impedances in Inductive Power Transmission Systems," Journal of Microwaves, Optoelectronics and Electromagnetic Applications, vol. 18, no. 4, pp. 492 - 504, 2019.

[13] D. C. Hamill, "Lumped Equivalent Circuits of Magnetic Components: The Gyrator-Capacitor Approach," IEEE Transactions on Power Electronics, vol. 8, no. 2, pp. 97 - , 1993.

[14] J. A. B. Faria and M. P. Pires, "Theory of Magnetic Transmission Lines," IEEE Transactions on Microwave Theory and Techniques, vol. 60, no. 10, pp. 2941-2949, 2012.

[15] R. F. Harrington, Time-Harmonic Electromagnetic Fields, Piscataway, NJ: Wiley-IEEE Press, 2001, pp. 61-66.

[16] C. A. Balanis, Antenna Design Theory, Hoboken, New Jersey: John Wiley \& Sons, Inc, 2005.

[17] R. M. Duarte and G. K. Felic, "Analysis of the Coupling Coefficient in Inductive Energy Transfer Systems," Active and Passive Electronic Components, vol. 2014, Article ID 951624, 6 pages, 2014.

[18] J. Gao, "Traveling Magnetic Field for Homogeneous Wireless Power Transmission," IEEE Transactions on Power Delivery, vol. 22, no. 1, p. 507, 2007. 